\title{
APLICACIÓN DE TECNOLOGÍA ANALÍTICA DE PROCESO (PAT) PARA CONTROLAR ATRIBUTOS DE CALIDAD CRÍTICOS UTILIZANDO LA ESPECTROSCOPIA NIR EN UN PROCESO DE FABRICACIÓN DE UN POLVO PARA SUSPENSIÓN
}

\author{
Ruben Cueva Mestanza*a
}

\begin{abstract}
RESUMEN
El trabajo define los pasos a seguir para determinar los atributos de calidad críticos en un proceso de fabricación de un producto farmacéutico. Analizándose el impacto y la criticidad de cada una de las operaciones de fabricación en la calidad del producto y definiendo los atributos críticos a controlar. De acuerdo con los resultados obtenidos, se decidió aplicar la espectroscopia NIR como alternativa a los métodos convencionales para controlar, de forma rápida, los atributos críticos durante el proceso de fabricación de un polvo para suspensión. El objetivo es construir la calidad del producto final, controlando atributos críticos de calidad de los productos intermedios en el mismo proceso aplicando la Tecnología Analítica de Procesos (PAT).
\end{abstract}

Palabras clave: control de calidad, atributos críticos de calidad, espectroscopia NIR, Tecnología Analítica de Procesos (PAT).

\section{APPLICATION OF PROCESS ANALYTICAL TECHNOLOGY (PAT) TO CONTROL CRITICAL QUALITY ATTRIBUTES USING NIR SPECTROSCOPY IN A POWDER MANUFACTURING PROCESS FOR SUSPENSION}

\begin{abstract}
In the present work are defined the steps to follow in order to determine the critical quality attributes in a manufacturing process of a pharmaceutical product. Analyzing the impact and criticality of each of the manufacturing operations in the quality of the product and defining the critical attributes to control. According to the results obtained, it was decided to apply NIR spectroscopy as an alternative to conventional methods to quickly control the critical attributes during the manufacturing process of a powder for suspension. The objective is to build the quality of the final product, controlling critical quality attributes of intermediate products in the same process by applying Process Analytical Technology (PAT).
\end{abstract}

Key words: Quality control, critical quality attributes, NIR spectroscopy, Process Analytical Technology (PAT).

*a Universidad María Auxiliadora. eduardo5121@hotmail.com 


\section{INTRODUCCIÓN}

La industria farmacéutica es reconocida como una de las más exigentes en cuanto al cumplimiento de estándares de calidad, considerando la criticidad de los productos que elabora. Sin embargo, a pesar de ello, frecuentemente se reportan eventos que comprometen la calidad de los medicamentos ${ }^{1}$, los que principalmente se encuentran asociados a la baja compresión del proceso y a la inadecuada estandarización de los procesos que obliga a la permanente actualización de la información reportada ante los organismos reguladores para lograr la autorización de comercialización del producto, lo que se traduce en costosas inversiones de recursos, tiempo y esfuerzo tanto para las empresas como para los organismos de control ${ }^{2}$. Por tal razón, durante los últimos años se han enfocado los esfuerzos hacia la búsqueda e implementación de estrategias basadas en la gestión del conocimiento y en la gestión del riesgo en calidad, teniendo en cuenta el aporte que significa su implementación.

Por otro lado, las nuevas normativas incluyen el concepto de Tecnología Analítica de Procesos (cGMP for the $21^{\text {st }}$ century publicada por la FDA $2002^{3}$ y EMA en el $2003^{4}$ ) como un sistema para el diseño, análisis y control de la producción farmacéutica a través de medidas de atributos críticos de calidad. Las medidas realizadas en las materias primas y durante el proceso o en parámetros del proceso con la finalidad de mejorar la calidad del producto final ${ }^{5}$. La aplicación de PAT es una necesidad debido a que las compañías farmacéuticas se enfrentan cada día a la creciente demanda de una mayor productividad y con menores costos de fabricación, además de cumplir con las necesidades de la evolución de los estándares de calidad, los cuales son cada vez más altos. El objetivo del PAT es mejorar el conocimiento y la comprensión del proceso para que los procedimientos se puedan realizar de manera diferente y sobre todo más eficiente, controlando durante el proceso atributos críticos. La aplicación de PAT nos permite (i) reducir el ciclo de producción a través de prueba de liberación rápida, (ii) mejorar consistentemente el proceso, (iii) reducir la pérdida de lotes o su reprocesamiento, a través de un aumento en el muestreo en tiempo real y promover la liberación de lotes en tiempo real. El objetivo de este trabajo es definir los atributos críticos en un proceso de fabricación y la aplicación de la espectroscopia NIR para evaluar la calidad del producto a partir de los atributos críticos: Secado y molienda (humedad), en la molienda y en la mezcla final (tamaño de partícula medio) y en el mezclado (homogeneidad), atributos que afectan directamente la calidad del producto final y de este modo alcanzar un mejor conocimiento del proceso y controlar cualquier desviación. Este trabajo se divide en dos partes. La primera consiste en examinar las etapas del proceso de fabricación y establecer los atributos críticos durante el proceso de fabricación de polvo para suspensión, la segunda corresponde a la aplicación de la espectroscopia NIR para controlar los atributos críticos de calidad: predecir humedad en el secado y la molienda, determinar el tamaño de partícula medio en la molienda y en la mezcla final, así como el punto final del mezclado, donde la mezcla se considera homogénea en la mezcla final en un polvo para suspensión. Estas metodologías son desarrolladas con el fin de ser aplicadas para entender y controlar el proceso. Aplicándose en el control de atributos críticos durante el proceso de fabricación de una forma farmacéutica sólida que se presenta como polvo para suspensión. 


\section{PARTE EXPERIMENTAL}

\section{Materiales y métodos}

1.- Revisión del proceso. El proceso de estudio involucra las etapas de dispensado o pesaje de las materias primas, molienda 1 (molienda de los componentes de la fórmula, excepto el principio activo y las esencias), mezcla, amasado, granulación, secado (secado con aire y secado con calor), molienda 2 (molienda de los componentes de la fórmula, excepto el principio activo y las esencias) y mezclado 2 (se adiciona el principio activo con las esencias), envasado y etiquetado, tal cual se muestra en la figura 1

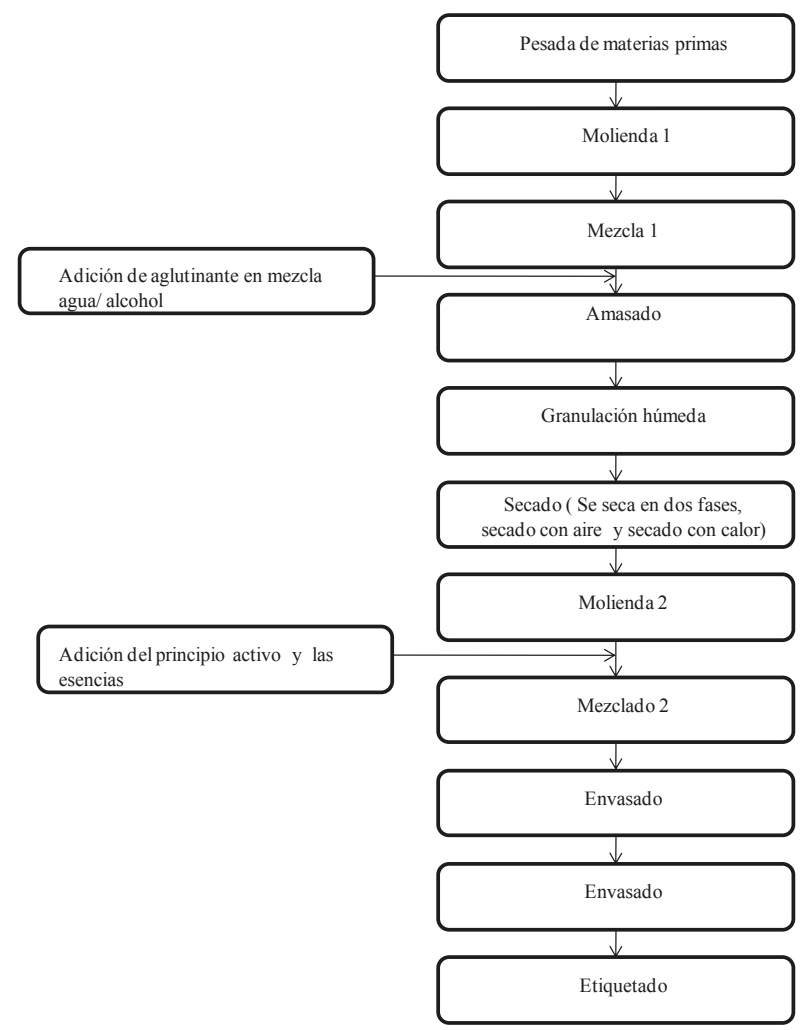

Figura 1. Etapas de la fabricación del producto polvo para suspensión.

\section{Definición de los atributos de calidad y los atributos críticos de calidad del producto}

Teniendo en cuenta que el producto polvo para suspensión ya se encuentra en el mercado, la definición de los atributos de calidad se realizó considerando la experiencia misma del laboratorio farmacéutico sobre el producto. Sobre esta base, fue realizado el análisis de cuáles de todos los atributos de calidad revestía particular criticidad, con el objeto de focalizar los esfuerzos de control de estos para garantizar su estricto cumplimiento. Los atributos críticos 
de calidad permiten focalizar los esfuerzos en el mejoramiento del producto y su proceso productivo. La tabla 1 muestra los resultados consensuados para evaluar la criticidad de los atributos críticos definidos a partir de los requerimientos técnicos del producto.

De otro lado, a cada atributo de calidad se le asignó una puntuación de 1, 3 ó 9, teniendo en cuenta el análisis previamente realizado acerca de la importancia de cada uno de ellos $\mathrm{y}$, fundamentalmente, su incidencia en el desempeño del producto y su aceptabilidad por parte de los consumidores. Como se observa, los mayores puntajes han sido otorgados al peso, humedad, potencia y a la uniformidad de contenido.

Tabla 1. Atributos críticos de calidad para el producto polvo para suspensión.

\begin{tabular}{|c|c|c|c|c|c|c|c|c|}
\hline 1 & 9 & 3 & 3 & 3 & 9 & 9 & 9 & 9 \\
\hline \multirow[t]{2}{*}{$\mathrm{pH}$} & Peso & \multicolumn{3}{|c|}{$\begin{array}{c}\text { Propiedades } \\
\text { organolépticas }\end{array}$} & $\begin{array}{c}\text { Volumen de } \\
\text { entrega }\end{array}$ & Potencia & Humedad & $\begin{array}{c}\text { Uniformidad de } \\
\text { contenido }\end{array}$ \\
\hline & & Color & Salor & Olor & & & & \\
\hline
\end{tabular}

Se realizó un análisis para identificar las etapas del proceso productivo que resultan críticas para el cumplimiento de las especificaciones de calidad del producto (tabla 2). De acuerdo con los resultados obtenidos, se identifican tres etapas del proceso de producción que resultan críticas para la calidad del producto: Secado, molienda 2, y mezcla 2 (valores superiores a 150 de ponderación).

Tabla 1. Matriz de ponderación de la criticidad de cada una de las etapas del proceso de fabricación del producto polvo para suspensión, en el cumplimiento de sus especificaciones.

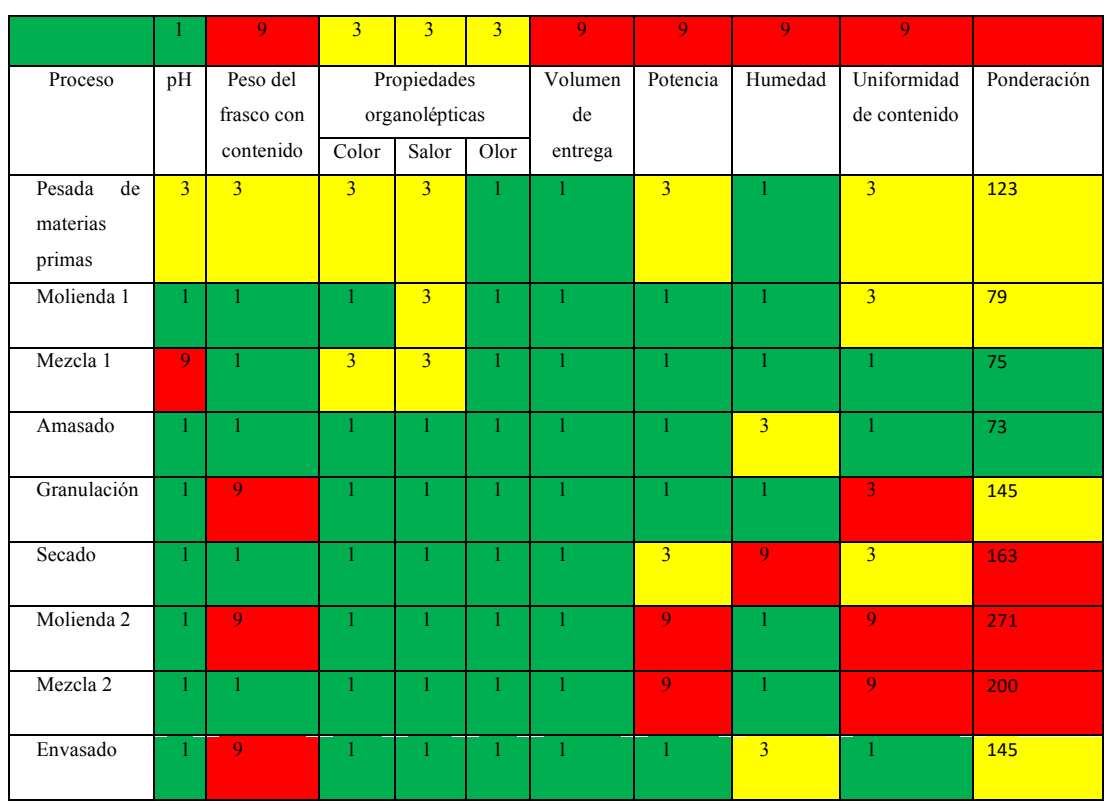


En estas tres etapas del proceso de fabricación de polvo para suspensión se determina, a partir de un análisis de riesgo, que los errores en los procesos afectan directamente la humedad, el tamaño de partícula y la homogeneidad (atributos críticos de calidad en los productos intermedios). Por tal razón, se seleccionaron estos atributos para ser analizados y controlados por una técnica rápida, como la espectroscopia NIR, durante el proceso de fabricación de polvo para suspensión.

Hardware y Software. Los espectros de reflectancia fueron adquiridos con un espectrofotómetro Analizador NIR de mano PHazir TM. (Polychromix, inc) controlado a través del PolychromixPhazirTM MG; cada espectro fue el resultado de 5 scans. Los espectros se registraron en un rango de longitudes de onda de 1600-2400 nm con una resolución de $12 \mathrm{~nm}$. El software Unscrambler V. 9.8 de CamoProcess (Trondheim, Norway) fue utilizado para el tratamiento matemático.

Registro de los espectros. Los espectros se registraron a través de la bolsa de plástico donde se recogió la muestra del proceso de mezclado, molienda y secado, en el un rango de 1600$2400 \mathrm{~nm}$, usando como referencia una placa de cerámica presente en la tapa de referencia del equipo.

Pre-tratamientos de los espectros NIR. Con el objetivo de reducir la variabilidad asociada a las características físicas y/o ruido de los espectros obtenidos de la fase de mezclado y secado, los espectros fueron sometidos a diferentes pre-tratamientos espectrales, como: Estándar Normal Variate (SNV), $1^{\text {era }}$ y $2^{\text {da }}$ derivada utilizando en estas últimas el algoritmo de SavitzkyGolay con una ventana móvil de tres puntos y ajustados a un polinomio de $2^{\text {do }}$ grado.

\section{Aplicación de la espectroscopia NIR para el control de atributos críticos de calidad}

\section{a) Determinación de la humedad}

Método de referencia. El método de referencia es el análisis por pérdida por secado en un analizador de Humedad Mettler Toledo Modelo HB43-S, se pesó alrededor de un gramo de muestra a una temperatura de $60^{\circ} \mathrm{C}$.

\section{Método NIR}

\section{Muestras para crear el método para cuantificar la humedad}

La ecuación de calibración PLS1 para cuantificar la humedad se estableció con muestras de producción (muestras de producción recogidas durante el proceso de secado). Se comprobó la humedad por el método tradicional/referencia (balanza de humedad). El conjunto de muestras se dividió en dos grupos. El primer grupo, conjunto de calibración, se empleó para construir el modelo de calibración. El segundo grupo, conjunto de validación, se utilizó para validar la ecuación de calibración PLS1 y determinar su capacidad predictiva.

\section{Modelo PLS}

El rango espectral empleado para construir el modelo de cuantificación seleccionado es entre 1869-1985 nm, ya que en este rango tiene mayor absorbancia el agua (figura 3). 
El pre-tratamiento matemático de los espectros seleccionados permite la corrección de la derivada de la línea base como consecuencia de la variabilidad habitual en las propiedades físicas de las diferentes muestras. El espectro NIR depende tanto de las propiedades químicas de la muestra como de algunas de sus propiedades físicas como son la forma, el tamaño de partícula o el grado de compactación de la muestra. El conjunto de muestras de calibración debe recoger todas las fuentes de variabilidad posibles, por ello son necesarias muestras de producción para incorporar al modelo la variabilidad debida al proceso de fabricación. Las muestras fueron recogidas durante el proceso de fabricación. El modelo ha sido validado, en primer lugar, internamente para asegurar su consistencia, usando espectros de muestras de producción. En este paso, se verifican y definen las longitudes de onda a emplear, el número de componentes PLS necesarios y otros parámetros del modelo PLS1. En segundo lugar, se ha efectuado la validación externa del modelo PLS1 empleando muestras de producción diferentes a las muestras empleadas para la validación interna.

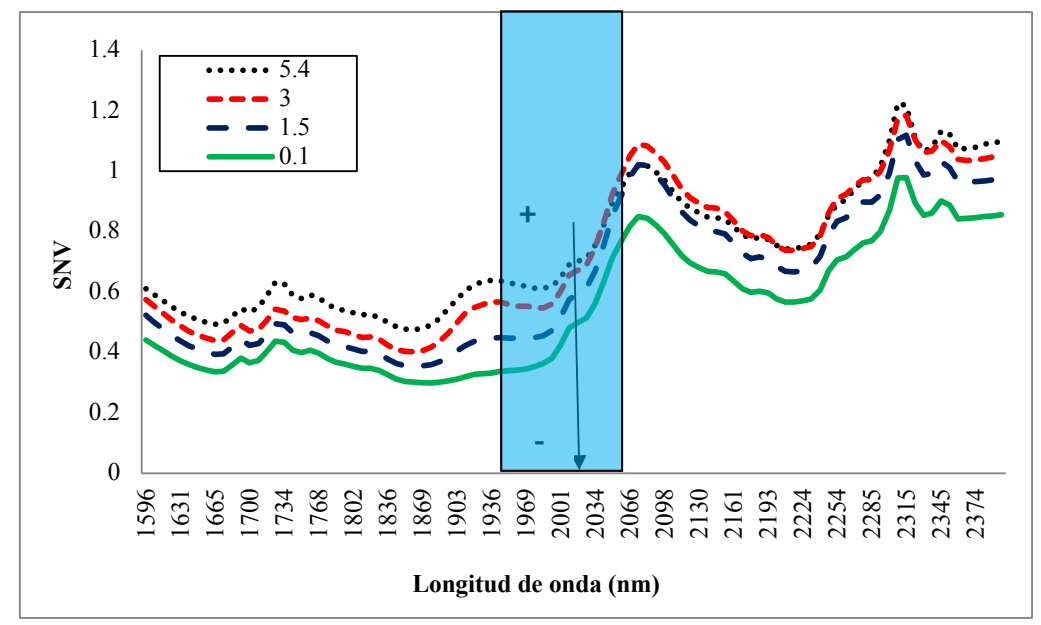

Figura 2. Espectros de muestras con distinto porcentaje de humedad.

El criterio para establecer el número de factores es seleccionar aquel que proporcione un menor error en predicción: RMSE (Root Mean Square Error) ha sido utilizado para evaluar los errores de calibración y predicción, definidos como RMSEC y RMSEP, respectivamente, seleccionando el número de factores en función del error de predicción.

n es el número de muestras, YiNIR corresponde a los valores predichos por el modelo y YiREF los valores de referencia. El RMSE puede ser considerado como el error medio obtenido en el proceso de modelado y está expresado en las mismas unidades que los datos de referencia. 
La tabla 3 muestra los parámetros más significativos del modelo PLS construido. Aunque el primer factor PLS describe un $98,1 \%$ de la varianza total, fue necesario tres factores $(98,5 \%)$ para ajustar correctamente el modelo y conseguir una buena capacidad predictiva. Los parámetros más relevantes del modelo se recogen en la tabla 3.

Tabla 3. Parámetros relevantes de los modelos de calibración construidos para la determinación de la humedad en polvo para suspensión.

\begin{tabular}{ccc}
\hline & \multicolumn{2}{c}{ Humedad } \\
\hline Muestras & Calibración & Predicción \\
Pre-tratamiento & Producción & Producción \\
Rango (nm) & SNV & SNV \\
Rango de humedad \% & $5.4-0.16$ & $\mathbf{1 8 6 9 - 1 9 8 5}$ \\
Factores & 3 & $0.17-2.3$ \\
Varianza explicada Y (\%) & 98.5 & \\
N $^{\circ}$ muestras & 23 & 35 \\
RMSEC/P (\%) & 0.21 & 1.37 \\
\hline
\end{tabular}

\section{b) Determinación del tamaño de partícula medio}

\section{Método de referencia}

Se determinó el tamaño de partícula medio de los granulados de producción, tomando una muestra de alrededor de $20 \mathrm{~g}$ de cada granulado y tamizándolo en cuatro fracciones de diferentes tamaños de partícula. Se seleccionaron tres tamices de 425, 250, $180 \mu \mathrm{m}$, además se recogió la fracción de granulado $<180 \mu \mathrm{m}$ que atravesó todos los tamices. Cada una de las fracciones se pesó en una balanza analítica, obteniéndose así el porcentaje de muestra en cada tamiz. Los datos fueron utilizados para obtener el tamaño de partícula medio según la fórmula siguiente [17].

$$
\text { Tamaño de partícula medio }=\frac{\text { Peso de la fracción Xno tamaño de la partícula de la fracción }}{\text { Peso total }}
$$

\section{Método NIR}

\section{Muestras para crear el método para cuantificar el tamaño de partícula medio}

La ecuación de calibración PLS1, para cuantificar el tamaño de partícula medio, se establece con muestras de producción (muestras de producción recogidas después de la molienda). Se comprobó el tamaño de partícula medio por el método tradicional/referencia (tamizado).

El conjunto de muestras fueron divididos en dos grupos. El primer grupo, conjunto de calibración, fue empleado para construir el modelo de calibración. El segundo grupo, conjunto de validación, se utilizó para validar la ecuación de calibración PLS1 y determinar su capacidad predictiva. 


\section{Modelo PLS}

El rango espectral empleado, para construir el modelo de cuantificación, se ha seleccionado entre 1595-1877, 1993-2284 nm, ya que en este rango el agua no influye en el espectro.

El espectro NIR depende tanto de las propiedades químicas de la muestra como de algunas de sus propiedades físicas, como son la forma, el tamaño de partícula o el grado de compactación de la muestra. El conjunto de muestras de calibración debe recoger todas las fuentes de variabilidad posibles, por ello son necesarias muestras de producción para incorporar al modelo la variabilidad debido al proceso de fabricación. Las muestras fueron recogidas durante el proceso de molienda 2 y mezcla 2.

El modelo ha sido validado, en primer lugar, internamente para asegurar su consistencia, usando espectros de muestras de producción. En este paso, se verifican y definen las longitudes de onda a emplear, el número de componentes PLS necesarios y otros parámetros del modelo PLS1. En segundo lugar, se efectuó la validación externa del modelo PLS1 empleando gránulos de la etapa de molienda 2 y mezcla 2 del producto polvo para suspensión.

El criterio para establecer el número de factores fue el mismo que se utilizó en el método para determinar la humedad.

La tabla 4 muestra los parámetros más significativos del modelo PLS construido. Fueron necesarios cinco factores $(90,2 \%)$ para ajustar correctamente el modelo y conseguir una buena capacidad predictiva. Los parámetros más relevantes del modelo se recogen en la tabla 4.

Tabla 4. Parámetros relevantes de los modelos de calibración construidos para la determinación del tamaño de partícula medio de polvo para suspensión.

\begin{tabular}{ccc}
\hline & \multicolumn{2}{c}{ Humedad } \\
Muestras & Calibración & Predicción \\
Pre-tratamiento & Producción & Producción \\
Rango (nm) & SNV & SNV \\
Rango de humedad \% & $5.4-0.16$ & $\mathbf{1 8 6 9 - 1 9 8 5}$ \\
Factores & 3 & $0.17-2.3$ \\
Varianza explicada Y (\%) & 98.5 & 35 \\
N $^{0}$ muestras & 23 & 1.37 \\
RMSEC/P (\%) & 0.21 & \\
& & \\
\hline
\end{tabular}


Toma de muestra. Se tomaron cuatro muestras a diferentes tiempos durante el proceso de mezclado con intervalo de 10 minutos, a los 10, 20 y 30 minutos, del inicio, a la mitad y al final de la descarga del mezclador en V. La figura 5 muestra los puntos donde se ha hecho el muestreo en el mezclador en $\mathrm{V}$.

\section{Método NIR}

Desviación estándar de espectros. Consiste en el cálculo de la desviación estándar de la absorbancia, DS, a cada longitud de onda de los espectros obtenidos a un determinado tiempo en el proceso de mezclado (Ec. 1), donde $n$ es el número de espectros ( $n=4), \bar{A}$ es el promedio de la absorbancia a cada longitud de onda de $\mathrm{n}$ espectros consecutivos y Aj es la absorbancia a cada longitud de onda de cada espectro $\mathrm{j}$.

$$
D S=\sqrt{\frac{\sum_{j=i}^{n}(\bar{A}-A j)^{2}}{n-1}}
$$

Después se calculó el promedio de DS para todas las longitudes de onda, según (Ec. 2), donde $\mathrm{m}$ es el número de longitudes de onda $\mathrm{i}$.

$$
\overline{\mathrm{X}}=\frac{\sum_{i=1}^{\mathrm{m}} \mathrm{S}}{\mathrm{m}} \text { (Ec. 2) }
$$

\section{RESULTADOS Y DISCUSIÓN}

1.- Determinación de la humedad. Se analizaron muestras después del secado, por el método NIR y por el método de referencia (balanza de humedad). Las muestras analizadas corresponden a 9 lotes. Por cada lote se tomaron cuatro muestras correspondientes a las cuatro fracciones en las que se dividió el lote para la etapa de secado. La aplicación de una prueba $t$ (paired test significance) al $95 \%$ de confianza, sobre las diferencias entre los espectros NIR y los valores de referencia reveló que los resultados de los dos métodos no muestran diferencias significativas entre ellos.

Al no presentar diferencias entre el método NIR y el de referencia se decidió aplicar el método NIR para determinar la humedad en muestras correspondientes a tres etapas críticas, donde la humedad es un atributo crítico, las etapas son: molienda 1, secado y molienda 2 (4 muestras por etapa). La figura 4 muestra los resultados obtenidos por el método NIR para cada una de las etapas. Observándose en la etapa de molienda 1 (etapa después de la adición del aglutínate) la humedad se encuentra entre 4 y $6 \%$. Determinar la humedad en esta etapa nos permite controlar nuestro proceso, ya que valores superiores indicarían que se ha adicionado demasiado diluyente o aglutinante y valores inferiores nos puede indicar lo contrario. La etapa del secado se dividió en dos, una con aire para eliminar el alcohol y otra con calor para eliminar el agua. Se observa que en el secado con aire los valores tienen mayor desviación que en el secado con calor, donde todas las muestras tienen valores inferiores a $0,5 \%$ de humedad. Por otro lado, en la etapa de molienda 2 , existe un ligero incremento de la humedad de las muestras. Esta observación de incremento de humedad en la molienda 
2, nos puede orientar a pensar que el producto capta humedad en el proceso. Por lo cual es importante el correcto almacenamiento de producto para evitar un aumento en el porcentaje de humedad, el cual puede afectar las características del producto final.

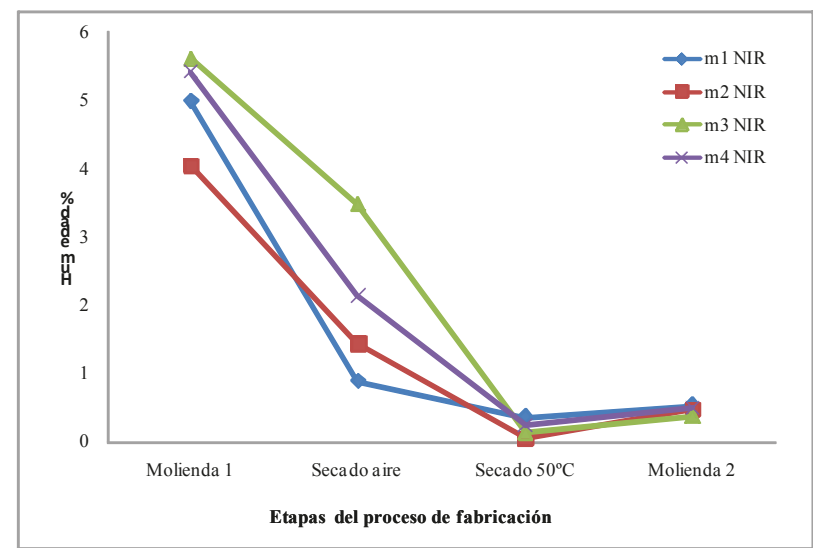

Figura 3. Evolución de los valores del contenido de humedad en tres etapas del proceso de fabricación de polvo para suspensión.

El método NIR, para determinar la humedad, se aplicó en la monitorización de 9 lotes, por cada lote se tomaron 4 muestras correspondientes a las cuatro fracciones en las que se dividió el lote para secarlo en el secador de lecho fluido. La figura 5 muestra los resultados obtenido por el método NIR para determinar la humedad. Probándose la aplicabilidad de este método para la monitorización de la humedad en procesos. Los resultados para los 9 lotes muestran que la etapa de secado está bajo control con resultados inferiores al límite ( $2 \%$ de humedad establecido para este producto).

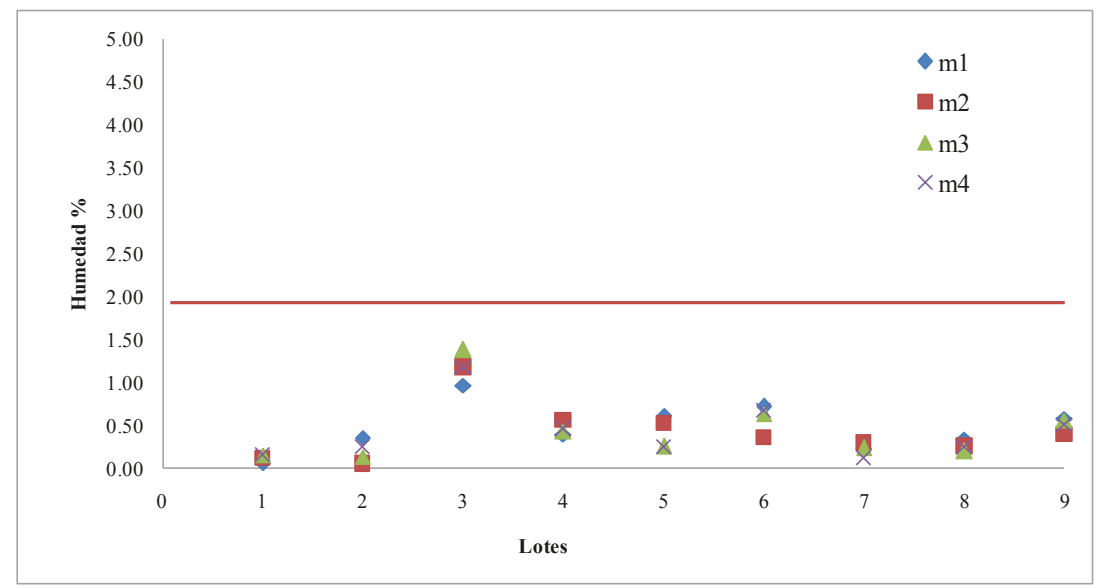

Figura 4. Evolución de los valores del contenido de humedad en la etapa de secado de 9 lotes consecutivos en un polvo para suspensión ( $2 \%$ de humedad máximo aceptable para el producto según las especificaciones). 
2.- Determinación del tamaño de partícula medio. Se analizaron muestras después de la molienda 2 y de la mezcla 2 por el método NIR y por el método de referencia (tamizado) estas muestras corresponden a 2 lotes, por cada lote se tomaron 4 muestras. La aplicación de una prueba $t$ (paired test significance) al $95 \%$ de confianza, sobre las diferencias entre los espectros NIR y los valores de referencia revelaron que los resultados de los dos métodos no muestran diferencias significativas entre ellos. El modelo construido se ha utilizado en la predicción del tamaño de partícula medio de 9 lotes en la etapa de la molienda 2, por cada lote se tomaron tres muestras correspondientes, al inicio, medio y final de la molienda: se ha obtenido una media de $214 \mu \mathrm{m}$.

La figura 6 muestra el gráfico de control de los tamaños de partícula medio predichos, donde observamos las fluctuaciones de tamaño de partícula medio de las muestras. Los valores se distribuyen aleatoriamente alrededor de un valor central (el promedio de los datos) y ninguno de los valores tiene un tamaño de partícula medio fuera del límite definido por el valor medio $\pm 2 \mathrm{DS}(\mathrm{DS}=$ desviación estándar).

El diagrama de control de los valores individuales es efectivo para controlar desviaciones y tendencias a partir de valores individuales y se puede deducir que el proceso de molienda 2 está bajo control.

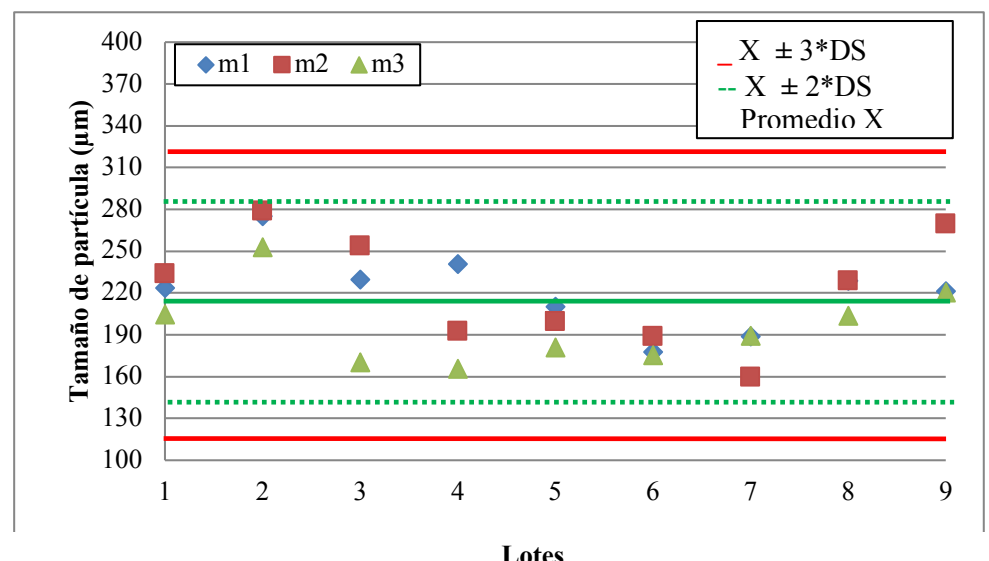

Figura 5. Gráfico de control de tamaño de partícula medio para muestras de la etapa de molienda 2 de polvo para suspensión.

\section{3.- Método cualitativo para determinar el punto final en el mezclado}

Determinación del pre-tratamiento. Para determinar los pre-tratamientos más idóneos se aplicaron varios tratamientos a cada uno de los espectros obtenidos con el espectrofotómetro NIR del proceso de mezclado, obteniéndose que el pre-tratamiento que reduce más la variabilidad física de la muestra y la producida por el sistema de registro, así como el ruido instrumental, fue Estándar Normal Variate (SNV). 
Determinación cualitativa de la homogeneidad de la mezcla. Previo a la aplicación de la metodología de desviación estándar de los espectros para la determinación cualitativa de la homogeneidad, se ha comprobado experimentalmente la desviación estándar de una mezcla que se considera homogénea, donde las variaciones son debidas a las variaciones producidas por el registro de los espectro y por el equipo NIR, registrando los espectros de una muestra representativa de la mezcla (mezcla de la parte inicial, media y final de la descarga) que ha sido homogenizada manualmente. Se registró cuatro veces, colocando la bolsa de plástico en diferentes posiciones cada vez, obteniéndose un valor de desviación estándar de 0,040 para los espectros obtenidos. Este valor se toma como valor objetivo para determinar cuándo la mezcla es homogénea. La figura 7 muestra la representación gráfica de desviación estándar frente al tiempo de muestreo, nos permite determinar a qué tiempo se considera la mezcla homogénea, considerándose que a los 10 minutos la mezcla no ha logrado la homogeneidad, debido a que la desviación estándar de los espectros es superior de lo que se espera para una mezcla homogénea; por otro lado, la mezcla a los 20,30 minutos y en la descarga se pueden considerar homogéneas ya que sus valores están por debajo del valor objetivo (muestras en las que las diferencias de sus valores solo se deben al registros de los espectros).

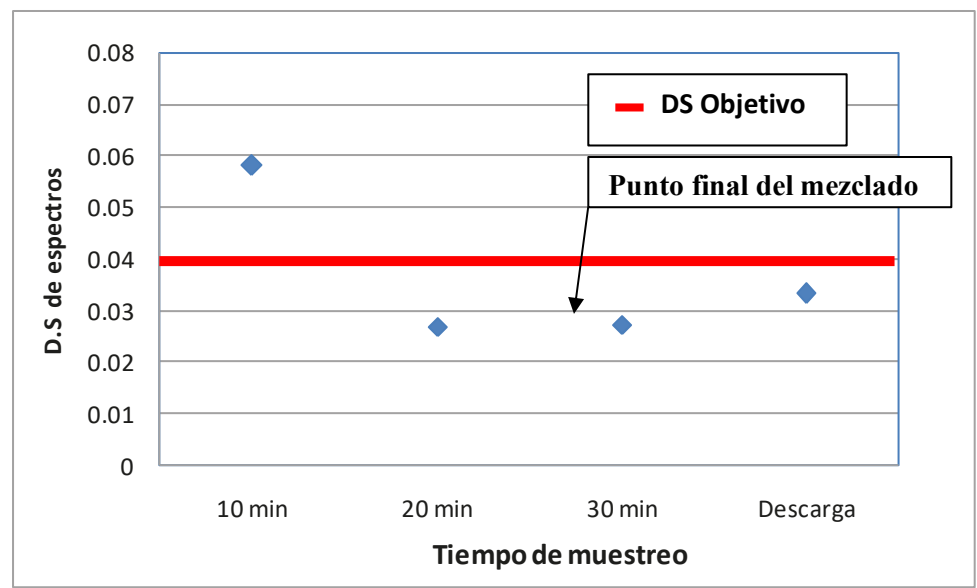

Figura 6. Aplicación de la metodología de la desviación estándar de los espectros a un proceso de mezclado de polvo para suspensión. 


\section{CONCLUSIONES}

A raíz de un estudio de análisis de riesgo se ha determinado los atributos que se tienen que controlar en la fabricación, los cuales son la humedad, el tamaño de partícula y la homogeneidad, para obtener un producto de calidad. Para poder controlar estos atributos críticos en el proceso, se ha aplicado NIR en el contexto de PAT. Desarrollándose nuevas metodologías para el seguimiento y control espectroscópico de un proceso de fabricación, los espectros obtenidos han permitido realizar un estudio cuantitativo de la humedad. El modelo de calibración PLS construido permite realizar la determinación correcta del contenido de humedad en el proceso de fabricación de polvo para suspensión. Se recomienda aplicar esta metodología en los procesos en que la humedad sea un factor crítico: productos higroscópicos, con principios activos inestables o que la humedad afecte la fluidez del granulado o la compactación. El modelo NIR aplicado permite determinar el tamaño de partícula medio en el proceso, el cual es un atributo crítico en el proceso de granulación, ya que de él depende la buena fluidez del polvo.

Este estudio demuestra la aplicación efectiva de NIRS para la determinación de la homogeneidad durante el proceso de mezclado, determinándose el tiempo final del mezclado a los 20 minutos. Se recomienda aplicar este método en fármacos, donde el mezclado es crítico en el proceso, por ejemplo, baja concentración del principio activo, componentes que se adhieren a las paredes, mezclas susceptible a desmezclado o segregación etc.

La espectroscopia NIR es una herramienta útil para determinar atributos críticos en el proceso de una forma farmacéutica.

\section{AGRADECIMIENTOS}

A Laboratorios Medifarma S.A y al D.T Q.F. Luis Kanashiro, por brindarnos todas las facilidades para la elaboración de esta investigación, cuyos resultados serán aplicados en la empresa. Por otro lado, quisiéramos brindar un agradecimiento especial al área de validaciones de procesos, representada por el Q.F. David Panta y Q.F. Erik Cotera, por su apoyo científico y técnico para entender mejor los procesos. 


\section{REFERENCIAS BIBLIOGRÁFICAS}

1. FDA. Warning Letters and Notice of Violation Letters to Pharmaceutical Companies [Internet]. Food and Drug Administration [citado 6 de febrero de 2018]. Disponible en: https://www.fda.gov/Drugs/ GuidanceComplianceRegulatoryInformation/EnforcementActivitiesbyFDA/ WarningLettersandNoticeofViolationLetterstoPharmaceuticalCompanies/default.htm

2. Yu LX. Pharmaceutical quality by design: Product and process development, understanding, and control. Pharm Res. 2008; 25(4): 781-791.

3. Watts DC. PAT. Paper presentado en: Heidelberg PAT Conference, 2006; Heidelberg, Alemania.

4. http://www.ema.europa.eu/ema/idex.jsp?curl=pages/regulation/document_listing/ document_listing_000162.jsp\&mid=WC0B01ac0580027089\#section1 (NO EXISTE LINK, ACTUALIZAR Y PONER LA INFORMACIÓN EN FORMATO INTERNET, VER EJEMPLO REFERENCIA 5)

5. FDA. Guidance for Industry PAT - A Framework for Innovative Pharmaceutical Development, Manufacturing, and Quality Assurance. [Internet]. Food and Drug Administration; 2004. [citado 20 noviembre de 2017]. Disponible en: http://www. fda.gov/downloads/Drugs/GuidanceComplianceRegulatoryInformation/Guidances/ ucm070305.pdf 